\title{
Como identificar as qualidades do desenho urbano por meio de uma matriz de análise para o ambiente construído
}

\author{
How to identify urban design qualities through the matrix analysis of built \\ environments
}

Drielle Vargas Nunes, David Sousa Vale

Universidade de Lisboa (ULisboa), Faculdade de Arquitetura, Lisboa, Portugal

\section{Resumo}

As qualidades do desenho urbano apresentadas pelo ambiente construído, importantes para quem se desloca a pé, são a imaginabilidade, a legibilidade, o enclausuramento, a transparência, a escala humana, a harmonia, a coerência, a complexidade e a organização. Por meio dessa premissa, conceitualiza-se um modelo que possibilita a leitura do ambiente construído de tal forma que possam ser descritas e medidas quais as qualidades presentes. Com a observação da rua, são caracterizados os aspectos físicos e funcionais encontrados, e, com a junção dessas características do ambiente construído, é possível identificar quais as qualidades existentes no local. Como caso de estudo foram selecionadas 91 ruas da cidade de Lisboa por meio de critérios eliminatórios e classificatórios. Percebe-se, assim, a grande diversidade de elementos apresentados dentro de um mesmo conjunto de ruas semelhantes, os quais fazem com que as qualidades apresentadas nas ruas sejam muito diversificadas, mesmo que os critérios de seleção tenham sido semelhantes. Para o urbanista, essa pode ser uma ferramenta de análise dos aspectos que influenciam a presença ou a ausência de determinadas qualidades no espaço canal, sendo possível proceder de forma consciente na qualificação dos espaços para torná-los mais adequados aos peões.

Palavras-chave: Qualidades do desenho urbano. Ambiente construído. Rua. Mobilidade pedonal. Lisboa.

\section{Abstract}

The qualities of urban design introduced by built environments that are important for foot travels are the imaginability, legibility, enclosure, transparency, human scale, linkage, coherence, complexity and tidiness. This premise contextualizes a model that enables the reading of built environments in such a way that the qualities present in them can be described and measured. With the observation of a street, its physical and functional aspects are characterized and, with the combination of these characteristics of the built environment, it is possible to identify what qualities exist in the place. As a case study, 91 streets in the city of Lisbon were selected through eliminatory and classificatory criteria. It was possible perceive the great diversity of elements present in the same set of similar streets. These elements make the qualities present in the streets very diverse,

DVN é licenciada em Arquitetura e Urbanismo, Estudos Avançados em Urbanismo, e-mail: driellevargas@gmail.com DSV é licenciado em Geografia e Planejamento Regional, doutorado em Architecture, Planning and Landscape, e-mail: david.vale@campus.ul.pt 
although the selection criteria were similar. For the urban planner, this can serve as an analytical tool of aspects that influence the presence or absence that certain qualities in the channel space, being possible to proceed consciously on the qualification of spaces to turn them more suitable for pedestrians.

Keywords: Urban design qualities. Built environment. Street. Pedestrian mobility. Lisbon.

\section{Introdução}

Que “[...] o espaço construído pelo homem pode aperfeiçoar a sensação e a percepção humana [...]" (Tuan, 1983, p. 114), isso já está claro. Entretanto, como é que o homem, o arquiteto e o urbanista podem refletir essa nova experiência no ambiente construído? "Estruturar e identificar o meio ambiente é uma atividade vital de todo o animal" (Lynch, 1997, p. 11), e, para tanto, a renovação se faz necessária quando é preciso interpretar a expressividade de determinado local. É nesse sentido que a orientação espacial, exercida pela linguagem e transmitida à imagem mental, é um instrumento essencial para a concepção de um ambiente construído legível. Para Lynch (1960), a legibilidade possibilita o deslocamento mais acelerado, diminui a possibilidade de estar perdido, eleva o senso de segurança e, para além destes, possibilita uma estrutura organizada de referência. Essa estrutura organizada de utilização da cidade pode constituir caráter influenciador sobre as decisões e os conceitos fundamentados em sociedade.

Como diz Hall (1986, p. 205), “[...] praticamente tudo o que o homem faz e é está ligado à experiência do espaço [...]." Sabendo dessa relação, reação e conexão que o homem tem com o ambiente em que está inserido, entende-se a necessidade de reconsiderar os conceitos de composição do ambiente construído. Esse novo argumento encontra sua fundamentação ideológica sobre a forma como o homem, enquanto ator social e formador da cidade, capta, absorve e reage diante das características físicas e funcionais do ambiente construído. Nesse contexto, diversos autores trabalharam com as qualidades que o ambiente construído pode demonstrar, assim como Gardner et al. (1996), que, por meio dos cinco Cs (conectividade, conveniência, conforto, convivência e conspicuidade), identificaram os atributos desejáveis para promover o caminhar.

É nesse contexto que Cullen (1983) define a cidade, com sua concepção baseada no meio ambiente, sendo por esse motivo um produto composto por edifícios, trânsito, árvores e natureza, tecidos entre si por elementos que despertam sentimentos. Para reestruturar o esquema urbano, o autor atribui três formas de leitura da cidade: onde há o movimento que determina os pontos de vista e a imagem; depois há o local e o conteúdo, respectivamente, que é onde se está e o que há de características físicas nesse local. Contudo é aqui que se encontra a questão fundamental para Cullen (1983), a qual consiste em reorganizar esses itens em um novo padrão fundamentado na imaginação urbana e nos valores subjetivos que envolvem a pessoa e o ambiente à sua volta.

Observar-se-á, assim, a parte da mobilidade urbana que não pode ser analisada pelas ferramentas do sistema de informação geográfica ou por imagens de satélite, mas que é muito importante para melhorar a possibilidade de caminhar por meio da caracterização de pequenas intervenções locais. Já que as mudanças nas "[...] facilidades para sentar, linhas de paisagismo, velocidade de tráfego são mais fáceis de alterar do que a densidade, os usos territoriais ou os padrões de rua [...]" (Park et al., 2015, p. 530), observar esses pequenos detalhes da composição do ambiente construído pode auxiliar na otimização do espaço urbano sem a necessidade de grandes intervenções. Desse modo, o contributo da caminhabilidade em micronível para promover espaços amigáveis à deslocação em modo ativo é mais apto a fornecer resultados interessantes no que se refere à escala humana (Gehl, 2006) do que outros níveis de estudo. Entretanto, também é verdade que o estudo que se segue não se direciona à caminhabilidade de forma global, com todos seus indicadores e aspectos conceituais. 0 objeto deste intento é o espaço-canal e o modo como as pessoas o exploram a pé.

Pode-se então dizer que um percurso transitável e habitável é aquele que permite ao seu utente a possibilidade de compreendê-lo, de utilizá-lo e de ver refletido, em seus aspectos físicos, as suas experiências (Tuan, 1983) e a sua cultura (Hall, 1986). É este o objetivo principal do desenvolvimento deste trabalho, a observação e a descrição das características físicas e funcionais do espaço urbano refletidas nas 
qualidades do desenho urbano e sua inter-relação com o uso pedonal. Sendo assim, as qualidades do desenho urbano são resultado de um processo interativo em que é necessária a observação de dois aspectos, o percurso e o pedestre, para que, por meio das qualidades do desenho urbano e das reações individuais, sejam alcançadas características físicas e funcionais do ambiente construído mais adequadas aos seus utilizadores.

Para tanto, descreve-se, na Tabela 1, uma compilação da bibliografia consultada para compreender quais as características físicas e funcionais do ambiente construído são necessárias de observação para que se possa identificar determinada qualidade do desenho urbano. Essa tabela é uma síntese do que será desenvolvido nos próximos tópicos deste artigo. Por exemplo, pode-se perceber que tanto Gardner et al. (1996) quanto Gehl (2006) referem que o item funções e usos, que tem a ver com a convivência entre as pessoas, é importante para se compreender a imaginabilidade do local, assim como Ewing \& Handy (2009) e Lynch (1960) indicaram em sua bibliografia que os materiais e as cores presentes nos locais observados também são de grande importância para a imaginabilidade. E, assim por diante, está descrito nessa tabela a bibliografia consultada a respeito das características que influenciam cada qualidade.
Dessa forma, ao se compreender a importância do estudo do ambiente construído por meio de uma observação em um nível mais detalhado, tendo por base as ferramentas que já foram utilizadas em estudos anteriores e os conceitos já utilizados, viu-se necessário introduzir ao assunto um modelo de análise do ambiente construído que possibilitasse a observação de cada qualidade do desenho urbano presente em determinado espaço e os itens que pudessem contribuir para que essa qualidade fosse otimizada ou melhorada.

\section{As qualidades do desenho urbano e suas características físicas e funcionais}

A rua como palco do caminhar diário e como componente de um percurso será descrita a seguir a partir de nove de suas qualidades urbanas, caracterizadas como imaginabilidade, legibilidade, enclausuramento, escala humana, transparência, harmonia, complexidade, coerência e organização. Essas qualidades foram estudadas e desenvolvidas ideologicamente por autores, como Lynch (1960), Jacobs (1993), Cullen (1983), Gehl (2006) e Hall (1986), e empiricamente no trabalho de Ewing et al. (2006) e Ewing \& Handy (2009). A proposta conceitual de

Tabela 1 - Relação entre as características físicas e as qualidades do desenho urbano

\begin{tabular}{|c|c|c|c|c|c|c|c|c|c|c|}
\hline Qualidades do desenho urbano & 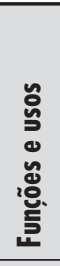 & 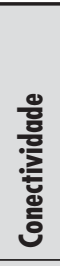 & 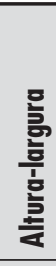 & 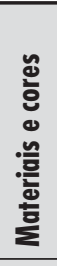 & 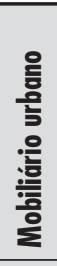 & 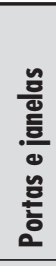 & 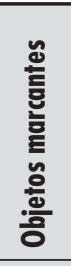 & 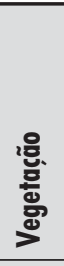 & 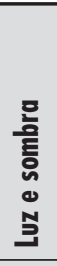 & 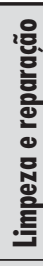 \\
\hline Imaginabilidade & $x$ & & & $x$ & $x$ & & $x$ & $x$ & & \\
\hline Legibilidade & $x$ & & & $x$ & $x$ & & $x$ & $x$ & & \\
\hline Enclausuramento & & & $x$ & $x$ & & & & $x$ & & \\
\hline Escala humana & $x$ & $x$ & $x$ & $x$ & $x$ & & & $x$ & & \\
\hline Transparência & & & & $x$ & & $x$ & & & & \\
\hline Harmonia & & $x$ & $x$ & $x$ & & $x$ & $x$ & & & \\
\hline Complexidade & $x$ & & & $x$ & & & $x$ & & $x$ & \\
\hline Coerência & $x$ & $x$ & & & & & $x$ & & & \\
\hline Organizaç̃óo & $x$ & & & $x$ & & $x$ & & & & $x$ \\
\hline
\end{tabular}

Fonte: do autor, com base em Cullen, (1983), Ewing \& Handy (2009), Gehl (2006), Jacobs (1961, 1993), Lynch (1960), Gardner et al. (1996). 
Ewing et al. (2006) e Ewing \& Handy (2009) é medir o que não pode ser medido, partindo de uma lista de 51 qualidades urbanas encontradas em referências bibliográficas anteriores. A partir da definição dessas 51 qualidades, o processo prossegue com a categorização e classificação delas por um painel de especialistas, o qual tem o dever de observar imagens e vídeos de diversas ruas, levando em consideração a importância das qualidades do desenho urbano para quem se desloca a pé. A partir dessa observação, foram selecionadas as nove qualidades mais significantes para quem se desloca a pé pela cidade de Lisboa.

É a partir dessas nove qualidades que será desenvolvido o estudo a seguir, lembrando sempre que

[...] estes elementos são apenas a matéria-prima da imagem do meio ambiente à escala urbana. Têm de ser trabalhados em conjunto de modo a conseguir uma forma satisfatória [...] (Lynch, 1997, p. 86).

Ou seja, o ambiente construído é analisado como um todo, entretanto constituído por partes importantes que, neste estudo, serão observadas separadamente. Para tanto, é preciso conceituá-las uma a uma e classificá-las a partir das características físicas presentes em cada uma delas, para, posteriormente, serem observadas diretamente em ambiente construído. Mas sabe-se ainda que, em pesquisas futuras, essas qualidades serão analisadas com as reações individuais das pessoas que utilizam as ruas estudadas.

\section{Imaginabilidade}

A imaginabilidade é definida como a "[...] qualidade de um objeto físico que lhe dá uma grande probabilidade de evocar uma imagem forte num dado observador [...]" (Lynch, 1997, p. 17). Não se refere apenas aos objetos, mas também àqueles lugares que denotam algum aspecto singular e próprio, sendo possível o seu reconhecimento ou a sua distinção em relação a outros lugares. É consequentemente atrativo e faz com que o observador desenvolva sentimentos e uma impressão duradoura e memorável em relação ao local.

Lynch (1960), em seu estudo das três cidades (Boston, New Jersey e Los Angeles), percebeu que, apesar dos diferentes resultados encontrados nas três situações, havia alguns traços do meio ambiente que eram comuns à percepção da maioria dos entrevistados, como os espaços abertos, a vegetação, o sentido de deslocação das vias e os contrastes visuais. Declaradas como paisagens favoritas, podem-se citar as linhas de água e os espaços abertos.

Em contraponto aos aspectos que contribuem para a imaginabilidade, podem ser citados aqui alguns elementos que dificultam a obtenção de uma imagem satisfatória das cidades, como as confusões, os pontos flutuantes, as fronteiras mal definidas, os isolamentos, as quebras de continuidade, as ambiguidades, as ramificações e a falta de símbolos e diferenciações. De modo geral, elevar o nível de imaginabilidade é permitir a fácil identificação dos lugares e a sua estruturação visual (Lynch, 1960).

\section{Legibilidade}

A maior indagação aqui se refere ao modo como os sinais e símbolos da cidade devem ser pensados e dispostos de forma a evocar, na maioria das pessoas, uma imagem definida e de fácil compreensão. Nesse sentido, Lynch (1960) divide as pessoas em grupos, segundo a idade, o sexo, a cultura, a ocupação, o temperamento ou a familiaridade. Para ele, é dessa forma que os planejadores e urbanistas precisam agir, para que o maior número possível de pessoas possa desfrutar satisfatoriamente do ambiente urbano. Outro aspecto relevante a se considerar ao pensar nos elementos presentes no ambiente urbano é a tricotomia identidade, estrutura e significado. Esses três componentes se apresentam em conjunto quando um objeto é legível.

A legibilidade, com isso, pode ser definida como "[...] a facilidade com que as partes podem ser reconhecidas e organizadas numa estrutura coerente [...]" (Lynch, 1997, p. 10), em um processo em que um indivíduo pode facilmente se deslocar pela cidade para encontrar o seu destino com destreza e rapidez. Nesse contexto, um local confuso pode ser agradável desde que seja um ponto diminuto dentro de um total legível e compreensível. Para que a deslocação seja agradável, um indivíduo não pode sentir o perigo de se perder, de não conseguir imaginar uma forma de chegar ao seu destino dentro da cidade. 


\section{Enclausuramento}

O enclausuramento é a definição dos espaços urbanos como outdoor rooms (Cullen, 1983), ou seja, o enquadramento do espaço em uma moldura visual que pode ser obtido pela presença de limites visuais laterais, superiores e inferiores no espaço urbano observado. Com isso, pode-se dizer que “[...] os espaços externos são definidos e formados por elementos verticais, que interrompem a linha de visão do observador [...]" (Ewing \& Handy, 2009, p. 73) ${ }^{1}$. Contudo, segundo Jacobs (1993), não são apenas os limites verticais que causam essa sensação de enclausuramento, mas também os elementos presentes no piso e a quantidade de céu que fica exposta à visão do observador.

A sensação de enclausuramento pode ser obtida como a principal ferramenta de estímulo ao senso de localização e identificação do entorno, o que faz entender que as pessoas reagem de forma favorável aos limites, acreditando ser a constituição de algo seguro. Para além da dimensão visual, a altura dos edifícios e os espaços entre eles determinam a quantidade de incidência solar, vento e temperatura do ambiente, o que pode favorecer a criação de espaços mais confortáveis e habitáveis (Jacobs, 1993).

Nesse sentido, existem alguns aspectos do ambiente construído que contribuem para a obtenção de outdoor rooms, como a relação entre a altura dos edifícios e a largura da rua, os muros, as árvores, os espaços entre os edifícios e a quantidade de céu que pode ser observada. Entretanto, segundo Jacobs (1993), os elementos visuais que melhor transmitem para o ambiente construído a sensação de enclausuramento são as árvores, mais importantes até do que os próprios edifícios presentes na rua.

\section{Escala humana}

A escala humana diz respeito à dimensão, textura e articulação dos elementos físicos encontrados em meio urbano, e sua relação com as dimensões humanas é a integração também desses elementos com a velocidade em que as pessoas se deslocam (Ewing

\footnotetext{
${ }^{1}$ Tradução do autor, texto original: “[...] outdoor spaces are defined and shaped by vertical elements, which interrupt viewers' lines of sight."
}

\& Handy, 2009) e a própria distância interpessoal definida por Hall (1986) e utilizada por Gehl (2006).

Para Jacobs (1993), a escala humana tem a ver com a possibilidade de reconhecimento entre as pessoas, ou seja, existe a possibilidade de reconhecer as pessoas a partir de seu perfil distante ou por meio da identificação de alguns detalhes de expressão a partir de uma proximidade maior. Essas distâncias de identificação interpessoal influenciam a definição da escala humana em ambiente urbano, assim como o ângulo pelo qual as pessoas podem identificar as coisas sem distorções. Para esse autor, os objetos podem começar a ser percebidos com clareza com o ângulo de 27 graus, ou seja, na proporção de 1:2, sendo 1 de altura para 2 de distância. Quando o observador está a 18 graus (1:3) do objeto, ele domina a situação, compreendendo o objeto, mas ainda sofrendo influências do entorno. Já com o ângulo de 12 graus (1:4) do objeto, ou menos, o observador e o objeto fazem parte do mesmo entorno, e o objeto pode ser visto com mais detalhes.

A partir dessa definição de ângulos, Jacobs (1993) percebeu que, para o favorecimento da escala humana, a altura máxima dos edifícios, para uma rua de 22 metros, seria de aproximadamente 9,15 metros e a altura mínima, para uma rua de 15 metros, seria de aproximadamente 6,50 metros. Mas a percepção de um lado e de outro da rua são diferentes, já que a observação angular possui como variável principal a distância do observador para o objeto. É nesse sentido que Ewing \& Handy (2009) destacaram a importância da velocidade de deslocação, já que a quantidade de alterações sofridas pela distância entre observador e objeto influencia demasiadamente a percepção do observador, ou seja, quanto mais rápido uma pessoa se desloca, menor é a quantidade de detalhes e objetos que ela consegue observar.

\section{Transparência}

A transparência pode ser descrita pela possibilidade das pessoas de perceber o que acontece além do limite da rua, ou até mesmo a sugestividade de sinais de habitabilidade de locais não utilizados pode favorecer a transparêcia (Ewing \& Handy, 2009). "É permitido saber, mesmo que não possa ser visto, que há algo do lado 
de dentro" (Jacobs, 1993, p. 286) ${ }^{2}$. As janelas e portas são especialmente importantes para a transparência, mesmo que não sejam inteiramente de vidro. Não é o vidro que demonstra o uso, mas a simples existência da possibilidade do uso caracterizada por uma porta ou uma janela em bom estado de conservação.

Sendo assim, é interessante para a transparência que existam indícios de que há alguma atividade humana para além da borda da rua, para que o observador tenha um senso de habitabilidade, conforto e possível refúgio. Nessas proporções, as portas antecedidas por pequenos vestíbulos de entrada, como uma reentrância na fachada, proporcionam ao observador essas sensações de acolhimento em ambiente urbano (Jacobs, 1993).

\section{Harmonia}

A harmonia aqui pode ser caracterizada pelas conexões físicas e visuais entre os edifícios e os espaços públicos de uma rua. É a complementaridade que um elemento exerce sobre o outro (Ewing \& Handy, 2009). É o conjunto de características físicas de um edifício que o fazem demonstrar algum respeito pelas outras construções do seu entorno e pela rua como um todo, tais como os materiais utilizados, a cor, as linhas de cornija, o tamanhos dos edifícios, as aberturas das janelas e seus detalhes, as entrâncias, as varandas, os alpendres, as linhas de sombra e os detalhes, como molduras e calhas ou algerozes. Essas características do ambiente construído não precisam estar sempre presentes em todas as great streets, como diz Jacobs (1993), mas precisam ser suficientes para expressar a complementaridade com os outros edifícios e com a rua.

\section{Complexidade}

A complexidade se refere à riqueza visual de determinado local e é diretamente proporcional à variedade de elementos físicos apresentados ao observador em determinado intervalo de tempo. Desse modo, a complexidade é variável de acordo

\footnotetext{
${ }^{2}$ Tradução do autor, texto original: "They let you know, even if you cannot see, that something is inside."
}

com a velocidade em que o espectador a observa. Assim, para um motorista de automóvel, os elementos que contribuem para a complexidade são percebidos de uma forma; já para o pedestre, essa organização do espaço canal é percebida de outra maneira. Dependendo da velocidade em que o observador se desloca, a distância entre os elementos mais marcantes precisa ser adaptada para que possam ser notados. Por exemplo, a quantidade de edifícios, a diversidade arquitetônica, as componentes paisagísticas, o mobiliário urbano, as sinalizações e as atividades de sociabilização humana (Ewing \& Handy, 2009) são alguns dos itens que precisam ser observados quando for preciso compreender a complexidade de um local.

Uma grande referência a essa complexidade ou diversidade urbana se dá por Jacobs (1961). A autora descreve quatro elementos básicos que precisam existir em uma rua para que ela possua essa diversidade exuberante mencionada: 1 ) a necessidade de usos principais combinados; 2) a necessidade de quadras curtas; 3) a necessidade de prédios antigos; e 4) a necessidade de concentração. Para ela, esses elementos são essenciais para que ocorra intensidade de usos nas ruas e calçadas.

\section{Coerência}

Acoerência tem também a ver coma complementaridade entre os edifícios e a rua, entretanto, nesse caso, o foco é sobre a qualidade da construção e do projeto em si, sobre o envolvimento de um novo objeto no meio em que se insere por meio da perspectiva do profissonal que o projeta e do profissional que o constrói de forma coerente e adequada ao espaço disponível (Ewing \& Handy, 2009). Alguns dos aspectos do ambiente construído que são importantes de ser observados para perceber a coerência são: a complementaridade da escala, a tipologia e disposição dos edifícios, o paisagismo, o mobiliário urbano, os materiais e os demais elementos físicos presentes na rua. A manutenção do ambiente urbano não pode dar vida a uma cor apagada e não pode colocar no local certo a árvore que está no local errado. Para que esses ajustes sejam feitos, é preciso elevado nível de qualidade de desenho e acabamento dos detalhes construtivos (Jacobs, 1993). 


\section{Organização}

A organização se refere à limpeza de um local, é a manutenção e a reparação do espaço de uso comum. Um local desarrumado, em desordem e com necessidade de limpeza e reparação é um local em decadência, não atrativo e que repele a vida urbana. Em contrapartida, um lugar arrumado e bem cuidado convida as pessoas a entrar ou a fazer uso dele. Contudo, a organização não é feita apenas de limpeza e reparo, mas também da escolha de materiais de acabamento e de elementos construtivos que sejam de fácil manutenção.

Dessa forma, depois de perceber quais as características físicas essenciais a cada qualidade do desenho urbano, podem-se extrair da bibliografia consultada os itens a serem observados em ambiente construído, para que seja possível compor cada qualidade. Por exemplo, para que possam ser percebidas as funções e os usos do local de estudo, devem-se observar os lugares reservados ao estacionamento de automóveis, a dimensão dos passeios e das ciclovias, a existência de algum elemento que atrapalhe a visão do pedestre e a quantidade de esplanadas existentes - as funções e os usos são importantes para perceber a imaginabilidade, legibilidade, escala humana, complexidade, coerência e organização. Para a conectividade, é necessário observar a existência de paragens de transportes públicos e faixas de pedestres ou passadeiras, e assim por diante.

\section{Da bibliografia ao modelo de estudo: uma metodologia}

Com a ferramenta de estudo definida em mãos, foram analisadas 91 ruas da cidade de Lisboa, seguindo múltiplos critérios de exclusão de variáveis. Esses critérios estão definidos com base na obtenção de uma amostra de análise que possibilite a comparação das ruas entre si, para além da descrição delas a partir das qualidades que elas apresentam, visando à compreensão dos aspectos do ambiente construído presentes em cada uma delas e dos motivos pelos quais essas características físicas e funcionais influenciam as qualidades do desenho urbano.

Para a escolha dessas ruas, foram utilizados os seguintes critérios eliminatórios: a) ruas do $3^{\circ}$ nível, segundo o Plano Diretor Municipal de Lisboa (Lisboa, 2013), com as ruas da cidade divididas em cinco níveis, quais sejam, $1^{\circ}$ nível - Rede Estruturante, $2^{\circ}$ nível
- Rede de Distribuição Principal, 3o nível - Rede de Distribuição Secundária, 4⿳⺈ nível - Rede de Proximidade e 5o nível - Rede de Acesso Local; b) declive máximo de 5\% (Félix, 2012); c) espaços centrais e residenciais dos tipos A, B ou C, setores de zoneamento da cidade onde é permitida a coexistência entre os vários usos urbanos, desde que compatíveis com o uso habitacional segundo o Plano Diretor Municipal de Lisboa (Lisboa, 2013). Ainda assim foram utilizados alguns critérios de classificação, como: a) trechos de rua que representem as características principais de toda a rua; b) trechos de ruas com possibilidade de serem agrupadas a outras em uma classificação prévia; c) ruas de uso multimodal.

Dessa forma e seguindo os critérios descritos, foram selecionadas as 91 ruas (Figura 1) utilizadas para a operacionalização da metodologia. A partir da observação das características físicas existentes em cada uma dessas ruas, é possível perceber a classificação da rua de acordo com as qualidades do desenho urbano que ela apresenta.

Em cada uma dessas ruas, foi executada uma análise, como a demonstrada na Tabela 2, na qual são observados itens específicos apresentados nas ruas estudadas, como os listados na segunda coluna dessa Tabela 2. Esses itens específicos do ambiente construído foram depois agrupados para formar cada qualidade do desenho urbano. Dessa forma, cada item é um elemento influenciador de uma ou de várias qualidades do desenho urbano. Por exemplo, os lugares disponíveis para estacionamento e a dimensão dos passeios e ciclovias contribuem para a imaginabilidade, legibilidade, escala humana, complexidade, coerência e organização. 0 comprometimento da visão dos pedestres e a quantidade de esplanadas também são elementos influenciadores das mesmas qualidades descritas anteriormente, entretanto, quando se fala em paragens de transportes públicos e passadeiras ou faixa pedonal, estes últimos influenciam a escala humana, harmonia e coerência, e assim sucessivamente.

Essa Tabela 2 relaciona as qualidades do desenho urbano e os itens a serem analisados in loco. Sendo assim, estão assinalados os itens que precisam ser observados para a composição de cada qualidade. Essa caracterização foi feita da seguinte forma: propôs-se uma apresentação dos dados distribuída de forma binária, em que o valor de 1 foi obtido quando as características física ou funcional eram apresentadas como na forma ideal demonstrada na Tabela 3 e o valor de 0 era apresentado quando as características não se enquadravam nos valores mínimos e máximos compatíveis com a Tabela 3. Podem ser observados 


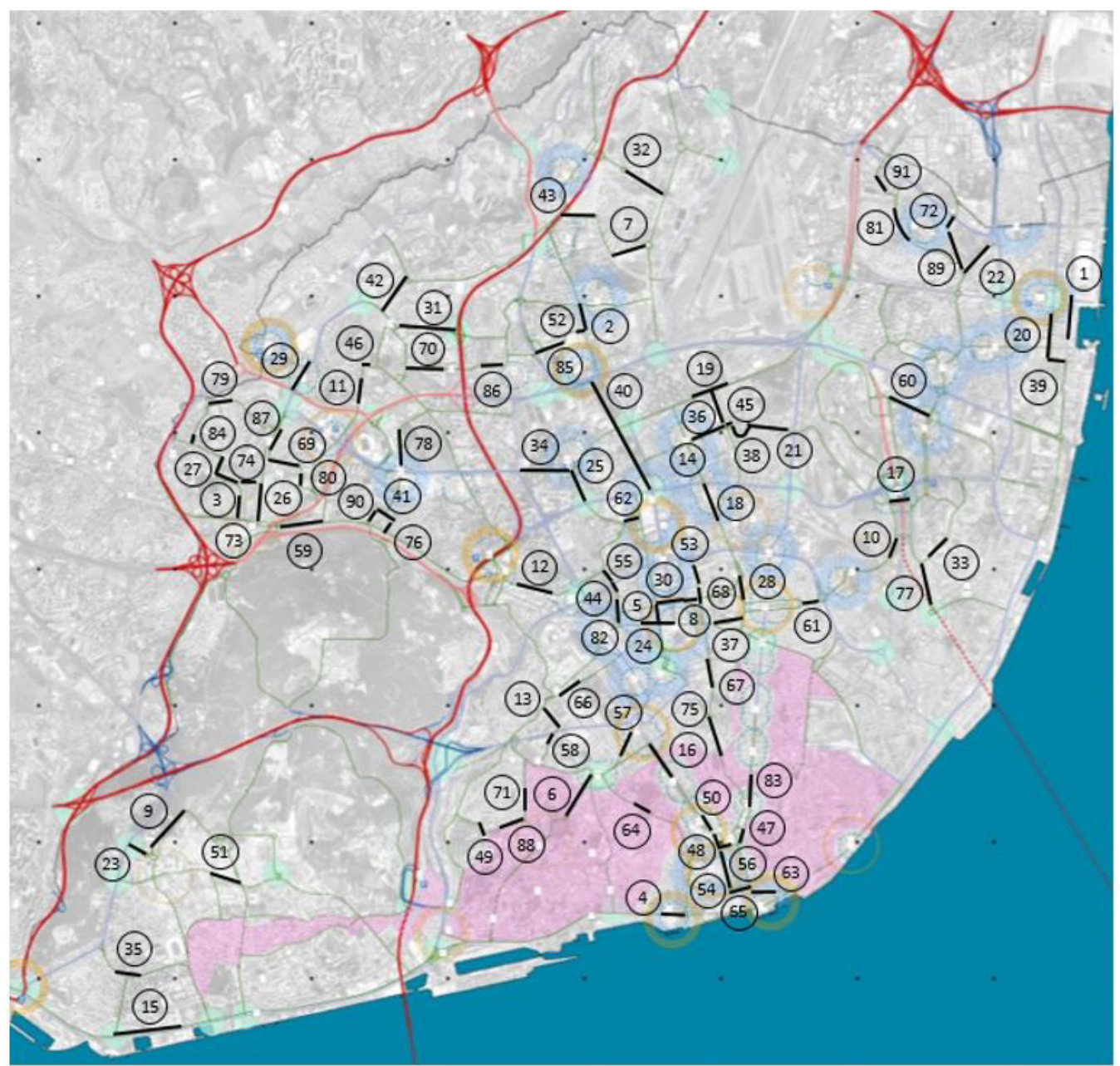

Figura 1 - 91 ruas da cidade de Lisboa

1-Alameda dos Oceanos

2-Alameda Linhas de Torres

3- Alameda Padre Álvaro Proença

4- Av. 24 de Julho

5-Av. 5 de Outubro

6-Av. Álvares Cabral

7-Av. Alvaro Cunhal

8- Av. Antônio José de Almeida

9- Av. Bombeiros

10-Av. Carlos Pinhão

11-Av. Colégio Militar

12-Av. Columbano Bordalo Pinheiro

13- Av. Conselheiro Fernando de Sousa

14- Av. da Igreja

15-Av. da Índia

16- Av. da Liberdade

17-Av. da Ucrânia

18- Av. de Roma

19-Av. do Brasil

20-Av. Dom João II

21-Av. Dom Rodrigo da Cunha

22-Av. Doutor Francisco Luís Gomes

23-Av. Doutor Mário Moutinho
24- Av. Duque Ávila

25-Av. Gama Pinto

26- Av. Gomes Pereira

27-Av. Grão Vasco

28-Av. Manuel da Maia

29- Av. Marechal Teixeira Rebelo

30-Av. Miguel Bombarda

31- Av. Nações Unidas

32- Av. Nuno Krus Abecassis

33-Av. Paulo VI

34-Av. Prof. Egas Moniz

35-Av. Restelo

36- Av. Rio de Janeiro

37-Av. Rovisco Pais

38- Av. Santa Joana Princesa

39- Av. Ulisses

40- Campo Grande

41- Estrada Benfica

42- Estrada Paço do Lumiar

43- Estrada Torre

44- Largo Azeredo Perdigão

45- Largo Frei Heitor Pinto

46- Largo Luz
47- Poço do Borratém

48- Praça Dom Pedro IV

49- Praça São João Bosco

50- Rua $1^{0}$ de Dezembro

51- Rua Actucenas

52- Rua António Stromp

53- Rua Arco do Cego

54- Rua Áurea

55- Rua Beneficência

56- Rua Betesga

57- Rua Braamcamp

58- Rua Carlos Alberto da Mota Pinto

59- Rua Carolina Michaelis de Vasconcelos

60- Rua Conselheiro Emídio Navarro

61- Rua Cristóvão Falcão

62- Rua Cruz Vermelha

63- Rua da Alfândega

64- Rua da Escola Politécnica

65-Rua do Comércio

66- Rua Dom Francisco Manuel de Melo

67- Rua Dona Estefânia

68- Rua Dona Filipa de Vilhena

69- Rua Doutor José Baptista de Sousa
70- Rua Fernando Namora

71- Rua Ferreira Borges

72- Rua Furriel Joc̃o Nunes Redondo

73- Rua Garridas

74- Rua General Morais Sarmento

75- Rua Gomes Freire

76- Rua Inácio de Sousa

77- Rua João César Monteiro

78- Rua João de Freitas Branco

79- Rua João Ortigão Ramos

80- Rua José Rodrigues Miguéis

81- Rua Loijstas

82- Rua Marquês Sá da Bandeira

83- Rua Palma

84- Rua Paz dos Reis

85- Rua Prof. Fernando da Fonseca

86- Rua Prof. Francisco Gentil

87- Rua Prof. Santos Lucas

88- Rua Saraiva de Carvalho

89- Rua Sargento Armando Monteiro Ferreira

90- Rua Sousa Loureiro

91- Rua Vice Almirante Augusto de Castro Guedes

Fonte: do autor. 
Tabela 2 - Itens analisados e as qualidades do desenho urbano

\begin{tabular}{|c|c|c|c|c|c|c|c|c|c|}
\hline & 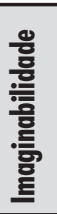 & 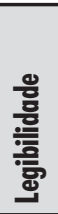 & 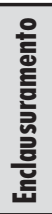 & $\begin{array}{l}\text { 름 } \\
\text { 롤 } \\
\text { 흘 } \\
\text { 랩 }\end{array}$ & $\begin{array}{l}\text { 른 } \\
\text { 힌 } \\
\text { 든 } \\
\text { 혼 }\end{array}$ & $\begin{array}{l}\text { 를 } \\
\text { 읕 } \\
\text { 호 }\end{array}$ & 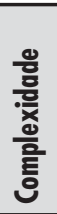 & $\begin{array}{l}\text { 른 } \\
\text { 흉 }\end{array}$ & 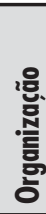 \\
\hline \multicolumn{10}{|l|}{ FUNÇÕES E USOS } \\
\hline 1. Disposiçõo dos lugares de estacionamento & $x$ & $x$ & & $x$ & & & $x$ & $x$ & $x$ \\
\hline 2. Extensão de lugares de estacionamento & $x$ & $x$ & & $x$ & & & $x$ & $x$ & $x$ \\
\hline 3. Dimensão dos passeios & $x$ & $x$ & & $x$ & & & $x$ & $x$ & $x$ \\
\hline 4. Dimensão das ciclovias & $x$ & $x$ & & $x$ & & & $x$ & $x$ & $x$ \\
\hline 5. Comprometimento da visão & $x$ & $x$ & & $x$ & & & $x$ & $x$ & $x$ \\
\hline 6. Quantidade de esplanadas & $x$ & $x$ & & $x$ & & & $x$ & $x$ & $x$ \\
\hline \multicolumn{10}{|l|}{ CONECTIVIDADE } \\
\hline 7. Passagem de autocarro & & & & $x$ & & $x$ & & $x$ & \\
\hline 8. Paragens de transportes & & & & $x$ & & $x$ & & $x$ & \\
\hline 9. Passadeiras & & & & $x$ & & $x$ & & $x$ & \\
\hline \multicolumn{10}{|l|}{ ALTURA E LARGURA } \\
\hline 10. Disposição dos edifícios & & & $x$ & $x$ & & $x$ & & & \\
\hline 11. Espaços entre edifícios & & & $x$ & $x$ & & $x$ & & & \\
\hline \multicolumn{10}{|l|}{ MATERIAIS E CORES } \\
\hline 12. Materiais de acabamento e revestimentos & $x$ & $x$ & $x$ & $x$ & $x$ & $x$ & $x$ & $x$ & $x$ \\
\hline 13. Cores empregadas & $x$ & $x$ & $x$ & $x$ & $x$ & $x$ & $x$ & $x$ & $x$ \\
\hline 14. Detalhes construtivos & $x$ & $x$ & $x$ & $x$ & $x$ & $x$ & $x$ & $x$ & $x$ \\
\hline 15. Material dos passeios & $x$ & $x$ & $x$ & $x$ & $x$ & $x$ & $x$ & $x$ & $x$ \\
\hline 16. Materiais e cores dos mobiliários & $x$ & $x$ & $x$ & $x$ & $x$ & $x$ & $x$ & $x$ & $x$ \\
\hline 17. Altura do lancil & $x$ & $x$ & $x$ & $x$ & $x$ & $x$ & $x$ & $x$ & $x$ \\
\hline \multicolumn{10}{|l|}{ MOBILLÁRIO URBANO } \\
\hline 18. Quantidade de bancos & $x$ & $x$ & & $x$ & & & & & \\
\hline 19. Quantidade de cinzeiros e lixeiras & $x$ & $x$ & & $x$ & & & & & \\
\hline 20. Quantidade de bancas e quiosques & $x$ & $x$ & & $x$ & & & & & \\
\hline \multicolumn{10}{|l|}{ PORTAS E JANELAS } \\
\hline 21. Quantidade de portas & & & & & $x$ & $x$ & & & $x$ \\
\hline 22. Existência de janelas, reentrâncias e pórticos & & & & & $x$ & $x$ & & & $x$ \\
\hline 23. Existência de entradas para estacionamento & & & & & $x$ & $x$ & & & $x$ \\
\hline 24. Quantidade de comércio com atividade & & & & & $x$ & $x$ & & & $x$ \\
\hline \multicolumn{10}{|l|}{ OBJETOS MARCANTES } \\
\hline 25. Existência de edifícios históricos & $x$ & $x$ & & & & $x$ & $x$ & $x$ & \\
\hline 26. Existência de edifícios identificáveis & $x$ & $x$ & & & & $x$ & $x$ & $x$ & \\
\hline 27. Existência de elementos marcantes & $x$ & $x$ & & & & $x$ & $x$ & $x$ & \\
\hline \multicolumn{10}{|l|}{ VEGETAÇÃO } \\
\hline 28. Quantidade das árvores & $x$ & $x$ & $x$ & $x$ & & & & & \\
\hline 29. Característica das árvores & $x$ & $x$ & $x$ & $x$ & & & & & \\
\hline \multicolumn{10}{|l|}{ LUZ E SOMBRA } \\
\hline 31. Quantidade de luz e sombra & & & & & & & $x$ & & \\
\hline 32. Existência de marquises, toldos e elementos de proteção & & & & & & & $x$ & & \\
\hline \multicolumn{10}{|l|}{ LIMPEZA E REPARAÇÃO } \\
\hline 33. Estado de conservação das portas e janelas & & & & & & & & & $x$ \\
\hline 34. Estado de conservação do mobiliário & & & & & & & & & $x$ \\
\hline 35. Estado de conservação do revestimento & & & & & & & & & $x$ \\
\hline 36. Limpeza da rua & & & & & & & & & $x$ \\
\hline 37. Vandalismo & & & & & & & & & $x$ \\
\hline
\end{tabular}

Fonte: do autor. 
Tabela 3 - Itens analisados em cada característica física

\begin{tabular}{|c|c|c|c|}
\hline $\begin{array}{l}\text { Características } \\
\text { físicas }\end{array}$ & Itens analisados & $\begin{array}{c}\text { Unidade } \\
\text { de medida }\end{array}$ & Descrição \\
\hline \multirow{6}{*}{ Funç̃oes e usos } & 1. Disposição dos lugares de estacionamento & $\mathrm{S} / \mathrm{N}$ & Pode ser longitudinal, perpendicular, paralelo - para resposta sim tem que ser longitudinal \\
\hline & 2. Extensão de lugares de estacionamento & $S / N$ & $\mathrm{E}<85 \%$ da extensão da rua \\
\hline & 3. Dimensũo dos passeios & $\mathrm{S} / \mathrm{N}$ & $1 / 6>P>1,50 m$ \\
\hline & 4. Dimensão das ciclovias & $\mathrm{S} / \mathrm{N}$ & 0 ideal é $1,50 \mathrm{~m}$ \\
\hline & 5. Comprometimento da visão & $\mathrm{S} / \mathrm{N}$ & Carros ou publicidade, ou outros próximos às passadeiras que atrapalhem a travessia da ruo \\
\hline & 6. Quantidade de esplanadas & $\mathrm{S} / \mathrm{N}$ & Mínimo 1/quadra \\
\hline \multirow{3}{*}{ Conectividade } & 7. Paragem de autocarro & $\mathrm{S} / \mathrm{N}$ & \\
\hline & 8. Paragens de transportes & $\mathrm{S} / \mathrm{N}$ & \\
\hline & 9. Passadeiras & $\mathrm{S} / \mathrm{N}$ & Nas esquinas \\
\hline \multirow{2}{*}{ Altura-Largura } & 10. Disposição dos edifícios & $\mathrm{S} / \mathrm{N}$ & 1:1,1 > ER > 1:2,5 - Relação entre altura do edifício e largura da rua \\
\hline & 11. Espaços entre edifícios & $\mathrm{S} / \mathrm{N}$ & Não haver espacosos vagos entre os edifícios \\
\hline \multirow{6}{*}{ Materiais e cores } & 12. Materiais de acabamento e revestimentos & $\mathrm{S} / \mathrm{N}$ & \\
\hline & 13. Cores empregadas & $\mathrm{S} / \mathrm{N}$ & \\
\hline & 14. Detalhes construtivos & $S / N$ & Homogêneos, elementos semelhantes empregados em toda a extensão da rua \\
\hline & 15. Material dos passeios & $S / N$ & \\
\hline & 16. Materiais e cores dos mobiliários & $\mathrm{S} / \mathrm{N}$ & \\
\hline & 17. Altura do lancil & $\mathrm{S} / \mathrm{N}$ & $5 \mathrm{~cm}<\mathrm{L}<10 \mathrm{~cm}$ \\
\hline \multirow{3}{*}{ Mobiliário urbano } & 18. Quantidade de bancos & $\mathrm{S} / \mathrm{N}$ & Mínimo de 1/100m \\
\hline & 19. Quantidade de cinzeiros e lixeiras & $S / N$ & Junto cos bancos \\
\hline & 20. Quantidade de bancas e quiosques & $\mathrm{S} / \mathrm{N}$ & Se houver \\
\hline \multirow{4}{*}{ Portas e janelas } & 21. Quantidade de portas & $\mathrm{S} / \mathrm{N}$ & $P>2 / 100 m$ \\
\hline & 22. Existência de janelas, reentrâncias e pórticos & $S / N$ & \\
\hline & 23. Existência de entradas para estacionamento & $\mathrm{S} / \mathrm{N}$ & Não haver \\
\hline & 24. Quantidade de comério com atividade & $\mathrm{S} / \mathrm{N}$ & $P>6 / 100 m$ \\
\hline \multirow{3}{*}{ Objetos marcantes } & 25. Existência de edifícios históricos & $\mathrm{S} / \mathrm{N}$ & \\
\hline & 26. Existência de edifícios identificáveis & $\mathrm{S} / \mathrm{N}$ & \\
\hline & 27. Existência de elementos marcantes & $\mathrm{S} / \mathrm{N}$ & \\
\hline \multirow{3}{*}{ Vegetação } & 28. Quantidade das árvores & $\mathrm{S} / \mathrm{N}$ & Mínimo de 1/100m \\
\hline & 29. Característica das árvores & $\mathrm{S} / \mathrm{N}$ & Para sim - as árvores precisam estar alinhadas e possuir porte e tamanho homogêneos \\
\hline & 30. Existência de arbustos, flores e vegetação & $\mathrm{S} / \mathrm{N}$ & \\
\hline \multirow[b]{2}{*}{ Luz e sombra } & 31. Quantidade de luz e sombra & $\mathrm{S} / \mathrm{N}$ & 50-50, aproximadamente. Para sim - ser observado uma média entre luz e sombra \\
\hline & $\begin{array}{l}\text { 32. Existência de marquises, toldos e elementos } \\
\text { de proteção }\end{array}$ & $\mathrm{S} / \mathrm{N}$ & \\
\hline \multirow{5}{*}{ Limpeza e reparaçãa } & 33. Estado de conservação das portas e janelas & $\mathrm{S} / \mathrm{N}$ & \multirow{4}{*}{$\begin{array}{l}\text { Para sim - o estado de conservaccão e a limpeza devem ser bons; não deve haver } \\
\text { sujeira e lixo na rua; e os edifícios e mobiliário precisam estar em bom estado }\end{array}$} \\
\hline & 34. Estado de conservação do mobiliário & $\mathrm{S} / \mathrm{N}$ & \\
\hline & 35. Estado de conservação do revestimento & $\mathrm{S} / \mathrm{N}$ & \\
\hline & 36. Limpeza da rua & $\mathrm{S} / \mathrm{N}$ & \\
\hline & 37. Vandalismo & $\mathrm{S} / \mathrm{N}$ & Não haver \\
\hline
\end{tabular}

Fonte: do autor. 
valores numéricos que correspondem à quantidade de vezes em que o número 1 é apresentado na matriz; assim, quanto mais vezes o número 1 aparecer, maior será a percentagem apresentada na última linha da matriz. Consecutivamente, quanto mais próximo de $100 \%$ os valores se apresentam, melhores são as condições em que as qualidades do desenho urbano se permitem ser observadas.

A Tabela 3 serve como base para a análise que será demonstrada no próximo tópico. Por meio dela, foram analisadas as ruas selecionadas para o caso de estudo e definidas, a partir dessa observação, quais as percentagens em que cada qualidade do desenho urbano aparece no local estudado.

Seguindo os parâmetros descritos anteriormente e sabendo que o caminhar será considerado nesse contexto como uma forma de transporte que merece ser inserido nas políticas de desenvolvimento dos transportes públicos e na promoção dos espaços públicos da cidade, mesmo que essa não seja uma realidade vertente (Gardner et al., 1996), introduz-se uma problemática que pode caracterizar, de forma substancial, a qualidade do ambiente construído, por meio desses mínimos elementos que podem ser redefinidos e reorganizados dentro do meio urbano. 0 ato de transitar por intermédio de modos ativos pela cidade pode ser aperfeiçoado e, muitas vezes, até mesmo encorajado. Segue-se, assim, a descrição dos resultados encontrados com essa análise do espaço canal e a consequente discussão a respeito do que pôde ser observado.

\section{Resultados e discussão}

Seguindo os critérios de escolha já definidos, este estudo iniciou-se com a quantidade de 200 ruas pertencentes ao $3^{\circ}$ nível hierárquico viário da cidade de Lisboa, de acordo com seu PDM (Lisboa, 2013). Após a aplicação de todos os critérios, foram encontrados 91 trechos de ruas, os quais foram então analisados seguindo o modelo de avaliação demonstrado na Tabela 3, na qual as características físicas e funcionais determinam diretamente quais as percentagens em que cada qualidade do desenho urbano é observada em cada rua.

Dessa forma, foram classificadas as 91 ruas de acordo com as características físicas que elas apresentaram. Na Tabela 4, constam apenas as percentagens finais de cada rua de acordo com as qualidades do desenho urbano que elas apresentam para que, assim, seja possível enquadrá-las em níveis para a seleção dos casos específicos para estudos futuros. Como pode ser observado na Tabela 4, existem quatro divisões. Esses intervalos englobam as ruas que possuem uma mesma faixa de classificação quanto às qualidades que possuem. Portanto, ficaram divididas as faixas de análise conjunta, conforme demonstrado na Tabela 5 , segundo o intervalo natural da amostra.

Além dessa observação individualizada de cada rua, as qualidades do desenho urbano também foram associadas ao estudo do potencial pedonal desenvolvido por Cambra (2012) na cidade de Lisboa, o qual leva em consideração os cinco Cs introduzidos por Gardner et al. (1996), que foram analisados em cada freguesia da cidade de Lisboa, como pode ser observado na Figura 2. As freguesias que apresentam os tons de rosa mais fracos tiveram também o potencial pedonal reduzido, enquanto que os tons de rosa mais fortes mostram a elevação desse índice de caminhabilidade, indicando que o local era mais adequado para quem se deslocava a pé.

Entretanto, para que este estudo se tornasse completo, viu-se necessário associar a esse índice de caminhabilidade as prováveis qualidades envolvidas do desenho urbano. Para tanto, as ruas estudadas individualmente foram agrupadas por freguesia, para que, com isso, fosse possível perceber a média das qualidades do desenho urbano em cada freguesia. Desse modo, percebe-se que há uma concentração maior de qualidades do desenho urbano justamente onde o potencial pedonal foi mais elevado. Contudo, observam-se algumas exceções, como a Ajuda e Alvalade, que apresentaram mais de $50 \%$ de qualidades do desenho urbano, mesmo que o potencial pedonal fosse mais reduzido, e a freguesia de Arroios, que, mesmo apresentando o potencial pedonal elevado, apresentou baixo índice de qualidades do desenho urbano.

Nesses casos, assim como o de Arroios, onde o potencial pedonal foi mais elevado, algumas alterações simples, como a locação de alguma vegetação, mobiliário urbano, esplanadas e reorganização dos lugares para estacionamento de carro, já elevariam as qualidades do desenho urbano. Casos específicos, como a Rua Gomes Freire, dentro da freguesia de Arroios, apresentaram uma quantidade reduzida de peões, mesmo estando enquadrada em uma região com boa caminhabilidade, e os motivos implícitos podem estar relacionados às qualidades que esses ambientes apresentam. 
Tabela 4 - Resultado da aplicação da Tabela 3

\begin{tabular}{|c|c|c|c|}
\hline Av. Carlos Pinhão & $18 \%$ & Av. Álvares Cabral & $44 \%$ \\
\hline Av. Bombeiros & $23 \%$ & Av. Miguel Bombarda & $44 \%$ \\
\hline Av. Rovisco Pais & $23 \%$ & Av. Rio de Janeiro & $44 \%$ \\
\hline Largo Azeredo Perdigão & $23 \%$ & Poço do Borratém & $44 \%$ \\
\hline Rua Gomes Freire & $23 \%$ & Alameda dos 0 ceanos & $45 \%$ \\
\hline Rua Sousa Loureiro & $24 \%$ & Av. Dom João II (até Av. Berlim) & $45 \%$ \\
\hline Av. Prof. Egas Moniz & $25 \%$ & Av. Nações Unidas & $45 \%$ \\
\hline Av. Alvaro Cunhal & $27 \%$ & Av. Nuno Krus Abecassis & $46 \%$ \\
\hline Rua Dom Francisco Manuel de Melo & $27 \%$ & Largo Luz & $46 \%$ \\
\hline Rua Paz dos Reis & $27 \%$ & Praça São João Bosco & $46 \%$ \\
\hline Rua João César Monteiro & $28 \%$ & Rua Cristóvão Falcão & $46 \%$ \\
\hline Av. Doutor Francisco Luís Gomes & $29 \%$ & Rua Beneficência & $47 \%$ \\
\hline Rua Conselheiro Emídio Navarro & $29 \%$ & Alameda Padre Álvaro Proença & $48 \%$ \\
\hline Estrada Torre & $30 \%$ & Av. Manuel da Maia & $48 \%$ \\
\hline Alameda Linhas de Torres & $31 \%$ & Campo Grande & $48 \%$ \\
\hline Av. Columbano Bordalo Pinheiro & $31 \%$ & Rua Braamcamp & $48 \%$ \\
\hline Av. Conselheiro Fernando de Sousa & $31 \%$ & Av. da Índia & $50 \%$ \\
\hline Rua General Morais Sarmento & $31 \%$ & Av. Gomes Pereira & $50 \%$ \\
\hline Av. 5 de Outubro & $32 \%$ & Rua Furriel João Nunes Redondo & $50 \%$ \\
\hline Av. Doutor Mário Moutinho & $32 \%$ & Rua Marquês Sá da Bandeira & $50 \%$ \\
\hline Rua João de Freitas Branco & $32 \%$ & Av. 24 de Julho & $51 \%$ \\
\hline Rua José Rodrigues Miguéis & $32 \%$ & Estrada Paço do Lumiar & $51 \%$ \\
\hline Rua Arco do Cego & $33 \%$ & Rua Ac̦ucenas & $52 \%$ \\
\hline Rua Prof. Santos Lucas & $34 \%$ & Rua Saraiva de Carvalho & $53 \%$ \\
\hline Av. Ulisses & $35 \%$ & Av. Gama Pinto & $54 \%$ \\
\hline Rua Prof. Fernando da Fonseca & $35 \%$ & Rua da Escola Politécnica & $54 \%$ \\
\hline Rua Cruz Vermelha & $36 \%$ & Rua João Ortigão Ramos & $54 \%$ \\
\hline Rua Lojistas & $36 \%$ & Rua $1^{\circ}$ de Dezembro & $56 \%$ \\
\hline Rua António Stromp & $37 \%$ & Rua da Alfândega & $56 \%$ \\
\hline Rua Garridas & $37 \%$ & Rua Fernando Namora & $56 \%$ \\
\hline Av. Marechal Teixeira Rebelo & $38 \%$ & Rua Sar. Armando Monteiro Ferreira & $56 \%$ \\
\hline Av. Paulo VI & $38 \%$ & Av. Grão Vasco & $57 \%$ \\
\hline Av. Restelo & $38 \%$ & Rua do Comércio & $59 \%$ \\
\hline Rua Inácio de Sousa & $39 \%$ & Rua Vice Al. Augusto de C. Guedes & $61 \%$ \\
\hline Av. da Ucrânia & $40 \%$ & Rua Betesga & $62 \%$ \\
\hline Estrada Benfica & $40 \%$ & Av. Colégio Militar & $63 \%$ \\
\hline Rua Doutor José Baptista de Sousa & $41 \%$ & Largo Frei Heitor Pinto & $63 \%$ \\
\hline Av. Santa Joana Princesa & $42 \%$ & Rua Ferreira Borges & $63 \%$ \\
\hline Rua Carlos Alberto da Mota Pinto & $42 \%$ & Rua Palma & $63 \%$ \\
\hline Rua Dona Estefânia & $42 \%$ & Av. Dom Rodrigo da Cunha & $64 \%$ \\
\hline Rua Prof. Francisco Gentil & $42 \%$ & Rua Áurea & $69 \%$ \\
\hline Av. de Roma & $43 \%$ & Av. António José de Almeida & $70 \%$ \\
\hline Av. do Brasil & $43 \%$ & Av. Duque Ávila & $72 \%$ \\
\hline Rua Carolina Michaelis de Vasconcelos & $43 \%$ & Praça Dom Pedro IV & $74 \%$ \\
\hline \multirow[t]{2}{*}{ Rua Dona Filipa de Vilhena } & $43 \%$ & Av. da Igreja & $81 \%$ \\
\hline & & Av. da Liberdade & $85 \%$ \\
\hline
\end{tabular}

Legenda. Classes referentes ao intervalo natural da amostra de acordo com Tabela 5.

Classe $1+$ Classe 2

Classe 3

Fonte: do autor.

urbe. Revista Brasileira de Gestão Urbana (Brazilian Journal of Urban Management), 2018 jan./abr., 10(1), 231-244 


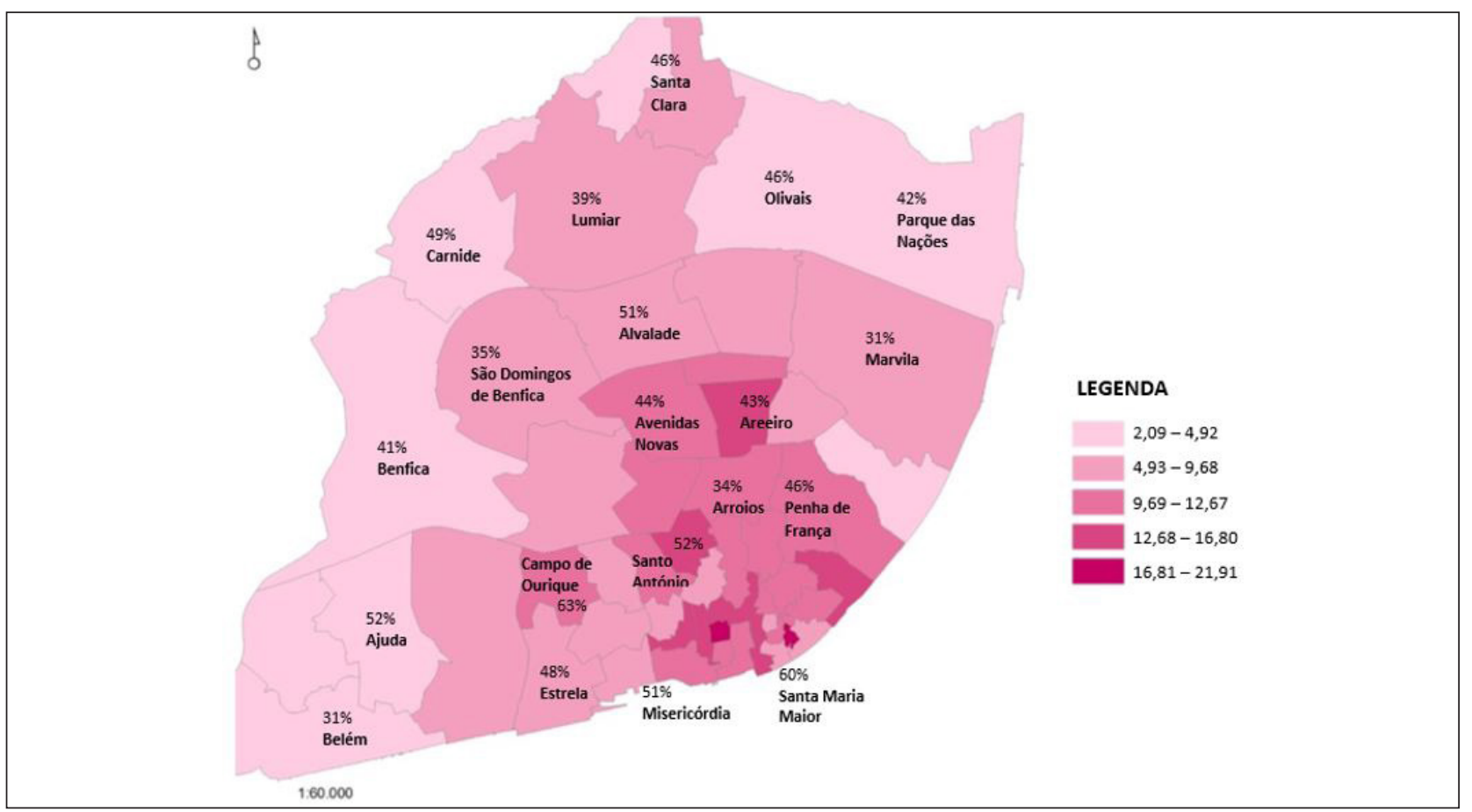

Figura 2 - Potencial pedonal de Lisboa por freguesia e as qualidades médias Fonte: Cambra (2012) e dados do autor.

Tabela 5 - Intervalo natural da amostra

\begin{tabular}{cccc}
\hline Classe & Mínimo & Máximo & $n$ \\
\hline 1 & 0,18122 & 0,359203 & 28 \\
2 & 0,37095 & 0,507127 & 39 \\
3 & 0,521268 & 0,63786 & 18 \\
4 & 0,688982 & 0,854358 & 6 \\
\hline
\end{tabular}

Fonte: do autor.

\section{Notas conclusivas}

Apesar de ser instintivo ao profissional que compõe, estrutura e reestrutura o ambiente construído, a observação das características físicas e a possibilidade de medição e quantificação desses itens podem caracterizar fator decisório ao se projetar ou reestruturar uma rua. Quando esses aspectos podem ser listados e observados em uma escala mais detalhada, as intervenções podem ser direcionadas aos itens que realmente podem melhorar o ambiente construído segundo a ótica de quem o utiliza por meio da mobilidade ativa.
Com isso, pode ser dito aqui que o processo de criação de espaços urbanos mais habitáveis e mais transitáveis passa por meio de diversas vertentes de projeto, inclusive no que se refere à qualidade urbana associada à atratividade de determinados locais. Nesse contexto, o estudo das qualidades do desenho urbano feito por intermédio das características físicas do espaço urbano pode constituir ferramenta necessária para perceber quais aspectos do ambiente construído precisam ser alterados para que esse local se torne mais habitável e mais transitável.

Observando de forma global, podem ser alcançados índices mais significativos em todas as 91 ruas estudadas, entretanto é preciso que o ambiente seja observado desde o seu pormenor, para que, com as pequenas intervenções locais, possam ser observados ambientes mais amigáveis aos peões. Por esse motivo foram introduzidos itens específicos a serem analisados no ambiente construído para caracterizar as qualidades do desenho urbano e, consecutivamente, apontar os itens que necessitam de intervenção para melhorar o desempenho de cada qualidade. Entretanto, é possível dizer que, para um trabalho futuro, será necessário desenvolver uma observação diferenciada, que atribua valores ou pesos a cada item analisado, pois, às vezes, pode-se perceber, 
pelo estudo já desenvolvido, que alguns itens possuem mais impacto na formação de uma qualidade do que outros. Nesse sentido, acredita-se que a introdução de uma análise de preferência declarada possa indicar com mais precisão esses valores.

Essa comparação entre o que é apresentado no potencial pedonal e nas qualidades do desenho urbano pode proporcionar uma perspectiva de observação mais satisfatória do espaço urbano para o profissional que desenvolve espaços mais adequados aos peões. Nesse sentido é que se recorreu à análise dos mapas sobrepostos, já que, dessa forma, podem ser observados os semelhantes resultados em relação ao potencial pedonal e às qualidades do desenho urbano. É possível dizer assim que o estudo dessas qualidades pode contribuir substancialmente para as análises da caminhabilidade e, ainda, complementá-lo, já que podem ser observados aspectos importantes para quem caminha em variadas escalas urbanas.

Dessa forma, este estudo é uma parte importante de um trabalho de tese que está sendo desenvolvido paralelamente, no qual é observado e mapeado também o comportamento das pessoas que fazem uso desses espaços, para que possa ser feita uma comparação entre as qualidades do desenho urbano observadas e a quantidade de pessoas que utilizam essas ruas e o modo como elas as utilizam. Além disso, será pesquisada a opinião dessas pessoas quanto ao seu vínculo com essas ruas, se as usam pela necessidade de estarem ali, pela opção ou por algum vínculo afetivo com o lugar.

\section{Agradecimentos}

À CAPES, pelo apoio a esta pesquisa, e ao CIAUD (Centro de Investigação em Arquitetura, Urbanismo e Design) da Faculdade de Arquitetura da Universidade de Lisboa.

\section{Referências}

Cambra, P. J. M. (2012). Pedestrian accessibility and attractiveness indicators for walkability assessment (Dissertação de Mestrado), Lisboa.

Cullen, G. (1983). Paisagem urbana. Lisboa: Edições 70.
Ewing, R., \& Handy, S. (2009). Measuring the unmeasurable urban design qualities related to walkability. Journal of Urban Design, 14(1), 65-84. http://dx.doi. org/10.1080/13574800802451155.

Ewing, R., Handy, S., Brownson, R. C., Clemente, O., \& Winston, E. (2006). Identifying and measuring urban design qualities related to walkability. Journal of Physical Activity \& Health, 3(Suppl 1), S223-S240. PMid:28834514. http://dx.doi.org/10.1123/jpah.3.s1.s223.

Félix, R. M. (2012). Gestão da mobilidade em bicicleta: necessidades, factores de preferência e ferramentas de suporte ao planeamento e gestão de redes. $O$ caso de Lisboa (Dissertação de mestrado). Universidade Técnica de Lisboa, Lisboa.

Gardner, K., Johnson, T., Buchan, K., \& Pharaoh, T. (1996). Developing a pedestrian strategy for London. Transport policy and its implementation. In Proceedings of Seminar B Held At The 24th European Transport Forum (Vol. P402). England: Brunel University.

Gehl, J. (2006). La Humanización del Espacio. Barcelona: Editorial Reverté.

Hall, E. T. (1986). A dimensão oculta. Lisboa: Relógio d’Água.

Jacobs, A. B. (1993). Great streets. Cambridge: MIT Press.

Jacobs, J. (1961). The death and life of great american cities. New York: Random House.

Lisboa. Câmara Municipal de Lisboa. (2013). PDM em vigor. Recuperado em 14 de outubro de 2013, de http://www.cmlisboa.pt/viver/mobilidade

Lynch, K. (1960). The image of the city. Cambridge: MIT Press.

Lynch, K. (1997). A imagem da cidade. Lisboa: Edições 70.

Park, S., Choi, K., \& Lee, J. S. (2015). To walk or not to walk: testing the effect of path walkability on transit users' access mode choices to the station. International Journal of Sustainable Transportation, 9(8), 529-541. http://dx.doi. org/10.1080/15568318.2013.825036.

Tuan, Y. (1983). Espaço e lugar: a perspectiva da experiência. São Paulo: Difel.

Recebido: Jan. 03, 2017

Aprovado: Maio 25, 2017 